\title{
MUJERES, POR MUJERES DE LA CIENCIA EN ARGENTINA
}

\section{Ester Elizabeth López Monrroy ${ }^{1}$}

\section{Resumen}

En Argentina seis de cada diez becaria/os del Consejo Nacional de Investigaciones Científicas y Técnicas (CONICET) son mujeres. Sin embargo, la mayoría femenina que se da al comienzo de la carrera se invierte notablemente al llegar a puestos jerárquicos, así del 75 por ciento inicial sólo quedan un 25 por ciento de mujeres. En consecuencia, la atribución de las categorías superiores no es neutral a la dinámica de género, pues la participación de la mujer en Ciencia, Tecnología e Industria reproduce las formas de opresión a la mujer que existen a nivel global; a tal punto que similares e, incluso, idénticas trayectorias académicas y profesionales son merituadas de un modo distinto si se es mujer o varón. Por ello, la taxonomía se concentra en las trayectorias de las cinco mujeres en comparación con las de los siete varones, que son los actuales directores de los Proyectos de Investigación Orientados (PIO) del Centro de Investigación y Transferencia de Catamarca (CITCA) por resolución 2049/16.

Palabras clave: Dimorfismo sexual, Género, CONICET y Argentina.

\section{INTRODUCCIÓN}

La idea de género se emplea para intentar explicar aquello que se entiende por femenino y masculino, en particular el carácter relacional de cada una de esas dos identidades. Así es que a lo largo de la historia, el determinismo biológico ha acudido al concepto de sexo para indicar el status de los sujetos, asumiendo el supuesto teórico de que es la genitalidad biológica la que determina las características y roles sociales. Sin embargo, los estudios de Stoller y Money en medicina, más precisamente el campo de la psicología muestran que hay algo más que el sexo y a ese algo más le llamaron género.

El sexo es asumido aún como un dato biológico inalterable, pero el énfasis esta puesto en la comprensión de lo femenino y lo masculino como identidades genéricas socialmente construidas. Hacia fines de los 80's y principios de los 90's, distintas teorías (Butler, 2001; Haraway, 1990) evidencian que son las mismas prácticas sociales las que construyen las diferencias sexuales, al convertir las diferencias promedio en diferencias categoriales.

Las teorías deconstructivistas retoman la noción foucaultiana de discurso (Butler, 2001), y proponen

\footnotetext{
1 Pós-Doutorada em Política de Inovação Inclusive do CITCA (CONICET UNCa), Catamarca, Argentina. E-mail: esterelizabethlopezmonrroy@gmail.com.
} 
que el género es un medio por el cual el sexo natural es producido y establecido como prediscursivo y anterior a la cultura (ESTÉBANÉZ, 2003: 5)

La construcción social de la femineidad y masculinidad forma parte inescindible de la fibra más íntima de la cultura de Argentina, pues se transmiten y subjetivan a través de la socialización. Se entiende así que determinadas actividades pertenecen al dominio exclusivo de una mujer o un varón. En 1994, la actual docente emérita de la Universidad de Buenos Aires (UBA) e investigadora del CONICET, Susana Torrado, salió al cruce de las declaraciones del entonces Ministro de Economía, Domingo Cavallo, por el feroz recorte presupuestario en el área de ciencia y tecnología. Sin embargo, lo llamativo del evento fue que Domingo Cavallo, en lugar de expresar los fundamentos que sustentaban la medida adoptada, la envió literalmente a lavar los platos. Expresión que evidencia que en su concepción de la organización social nacional el ámbito propio de una mujer es la cocina.

Todo lo cual implica la persistencia en Argentina de una idea limitada de la mujer, pues aquellas que, “... a comienzos del siglo XX, se animaron a transitar los claustros universitarios debieron sortear innumerables obstáculos, entre los que se cuentan la discriminación de recibir clases prácticas, en el caso de medicina, y hasta el título, en el de las parteras, en lugares diferentes a los utilizados por los varones" (VERA, 2015: 55). Pensamiento que parece no ser patrimonio exclusivo del país porque cuando la argentina, Paulina Luisi, intento ingresar a la Facultad de Medicina en Montevideo (Uruguay) le fue bastante difícil. Ya unos años antes, cuando a otra estudiante, Luisa Domínguez; se le negó la posibilidad de rendir exámenes de bachillerato, Antonio E. Vigil fundamentó la negativa en representación del Consejo Universitario diciendo: "Hacer cocido y hacer calceta, la olla y la aguja: he ahí el horizonte obligado de nuestras mujeres, cualquiera sea su posición social, sus tendencias y aptitudes" (VERA, 2015: 58)

La expresión de este pensamiento impregnado de un arcaísmo espeluznante puede parecer lejano, no obstante se encuentra bastante vigente. El encuentro de la Red del Norte y Nordeste sobre los Estudios de la Mujer y las Relaciones de Género (REDOR) en Brasil así lo demuestran, pues todas las investigaciones coinciden en que en el discurso del sistema patriarcal: la mujer posee un alma dominada por el útero, exceso de emocionalidad, el cerebro más liviano, las hormonas la domina, le falta testosterona para tomar decisiones, el lado izquierdo de su cerebro está más desarrollado y por eso habla más, pero es el lado derecho donde radica la capacidad matemática y el manejo espacial; en consecuencia no es buena para la matemática, física y las ciencias exactas, en general (MAFFIA, 2001: 33)

Minimización de la mujer que no tiene sustento científico alguno, pues las más destacadas investigaciones sostienen que:

“Eva y no Adán sería entonces el prototipo original humano... Se considera que el análisis de las 
capacidades en matemáticas permite valorar el nivel más elevado de la aptitud cognitiva. Importantes análisis efectuados en los Estados Unidos con grupos numéricamente muy significativos de jóvenes de ambos sexos, permiten afirmar en la actualidad que no existen diferencias genéricas en los resultados de las pruebas en matemáticas ni en el ulterior desarrollo académico (STEM fields: science, technology, engineering and mathematics) u ocupacional de los grupos analizados. El Coeficiente intelectual (IQ Binet Stern) es mayor en las mujeres específicamente en la capacidad para escribir, comprensión lectora, velocidad perceptual, memoria asociativa y habilidad verbal ... En relación con el mecanismo causal del dimorfismo femenino, se considera que la acción hormonal es mucho menos importante en los diferentes aspectos que lo definen" (ARRIGHI, 2012: 10)

"Hasta el momento no se han encontrado diferencias significativas entre la capacidad intelectual del hombre con respecto a la mujer. Lo que sí se sabe con certeza, al medir el cociente intelectual IQ es que entre los hombres el desvió estándar es mayor. Esto quiere decir que entre un determinado grupo de hombres en un extremo voy a encontrar a los que son muy brillantes, en el medio a la mayoría que son mediocres y en el otro a los que son casi tarados por así decirlo. En el caso de las mujeres esta variación no es tan evidente. Sin embargo, la media para ambos grupos da el mismo valor".

En idéntico sentido, "Sandra Witelson y sus colegas de la Universidad McMaster detectaron que la mujer posee gran densidad de neuronas en la parte de la corteza del lóbulo temporal que se asocia a la comprensión y producción del lenguaje. En el conteo de neuronas después de la muerte, los investigadores hallaron que de las seis capas presentes en la corteza, dos mostraban más neuronas por unidad de volumen en mujeres que en varones. Similar hallazgo fue posteriormente reportado en el lóbulo frontal. Dicha información permitió a los neurocientíficos explorar diferencias sexuales en el número de neuronas de manera correlativa con las habilidades cognoscitivas -examinando, por ejemplo, si el incremento de la densidad en la corteza auditiva femenina se relaciona con su mejor rendimiento en las pruebas de fluidez verbal (CAHILL, 2005: 42)

El conocimiento científico, pretendidamente "objetivo" en oposición a la subjetividad adjudicada a la mujer y, por lo tanto, tiene un fuerte sesgo de género. Además, la ubicación de esa subjetividad en un rango inferior y exclusiva de la mujer desconoce la naturaleza humana, pues por definición todo ser humano es racional, así como también posee en esencia sentimientos. En consecuencia, la enunciación de los problemas a investigar, los datos que pueden ser considerados válidos, las metáforas a partir de las que se construye el conocimiento, están marcados por ese sesgo. Máxime si se considera que mucho antes de saber se siente, se percibe que existe una pregunta e, incluso, cual es la respuesta a esa incógnita. Aún más, que ese conocimiento no se produce en abstracto, sino de manera situada, desde posiciones específicas en términos sociales y género. Por ello, es fácil ver 
desde la propia experiencia, que aunque pueda parecer diversa, tiene en común la posición sub alterna de la mujer en una sociedad patriarcal.

En este contexto, aunque los datos empíricos recientes revelan una intervención significativa de la mujer en la investigación científica de Argentina, esto es, su presencia es mayor (184) que la del varón (116) en menores de 36 años para el ingreso a Carrera de Investigador Científico y Tecnológico (CIC) de Temas Estratégicos del CONICET en 2017. Cabe aclara que esta línea de ingreso a la carrera prioriza la incorporación de investigadores, a través de una definición propia de prioridades en base al Plan Argentina Innovadora 2020 y, por lo tanto, se orienta a cubrir áreas de vacancia en relación con el conocimiento; excluyendo desde el inicio a las Ciencias Sociales y Humanas. Estas áreas consideradas prioritarias son: Salud, Desarrollo y Tecnología Social, Agroindustria, Ambiente y Desarrollo Sustentable, Tecnología, Tecnología y Tema Estratégico y Energía e Industria.

Los valores se invierten mínimamente en la Convocatoria de CIC para temas en general destinada a atender al desarrollo armónico de las distintas disciplinas, así la mujer presenta el 144 ingresos mientras que el varón obtuvo 157 en el 2017.

También es real que solo sucede al inicio de la carrera, pues este abordaje muestra la disminución de la diferencia al contemplar la categoría de los investigadores, así en el escalafón superior esa participación de la mujer se reduce sustancialmente (47) en comparación con la del varón (135) para diciembre del 2016. Ello, se explica porque en las primeras etapas la mujer se ha convertido en un insumo esencial por su laboriosidad y nivel de detalle, pero se la excluye de la decisión por el impacto que, supuestamente, podría tener su sensibilidad en el enfoque de determinadas cuestiones, en especial aquellas que están en relación con la honestidad en la administración de los recursos.

\section{OBJETIVOS}

En Argentina, el CONICET es la institución que nuclea a casi la totalidad de los investigadores en el país. Sin embargo, la primera mujer -y hasta el momento también la única- presidente del organismo nacional más importante fue la Doctora Marta Rovira en 2010, luego de cincuenta años desde la refundación institucional del CONICET. ${ }^{3}$ Marta es doctora en Ciencias Físicas, egresada de la Facultad de Ciencias Exactas y Naturales de la

\footnotetext{
${ }^{2}$ Entrevista personal realizada al biólogo, investigador del CONICET; Luís Oroño, 07 de marzo de 2018.

${ }^{3}$ El antecedente nacional inmediato lo constituye Consejo Nacional de Investigaciones Técnicas y Científicas (CONICYT) creado por decreto 9695/51 de Juan Domingo Perón y presidido por él mismo, que reunió a José Antonio Balseiro (Físico argentino, a quien se le solicitó especialmente que viniese de la Universidad de Mánchester, Inglaterra), Enrique Gaviola (Astrónomo) y Otto Gamba (Ingeniero Nuclear) cuya
} 
Universidad Nacional de Buenos Aires (UBA). Había sido directora del Instituto de Astronomía y Física del Espacio (1995-2005) y su especialidad se basa en el estudio del Sol. Ella sostuvo, en una entrevista concedida a Rosario Lufrano para Canal 7, que: "la mirada como mujer aporta un enfoque distinto" e, incluso, aseguró que en el país "hay científicos excepcionales en varias disciplinas". ${ }^{4}$

El estudio pretende develar cómo es la atribución de reconocimiento entre mujeres y varones al interior del CONICET, a partir del análisis de sus trayectorias académicas y profesionales. La respuesta tentativa a esta pregunta de base es que el reconocimiento difiere dependiendo si se es mujer o varón en la ciencia de Argentina.

En arras a lograr el objetivo general que conlleva este problema de investigación, se ha tomado una muestra representativa, esto es, el CITCA, pues su diversidad disciplinar, heterogeneidad en relación con la presencia de ambos sexos de trabajadores e información relevante como, por ejemplo, los datos del PIO permiten efectuar una aproximación más interesante que si solo se examinan los números como tales. Por ello, los objetivos específicos de trabajo son los siguientes:

Analizar la inserción, permanencia y promoción de la mujer en el Sistema Nacional de Innovación de Argentina, a través de la participación por género en la carrera del investigador científico para un caso concreto: el CITCA del CONICET;

comparar las trayectorias académicas y profesionales de las cinco mujeres en relación con las de los siete varones, que actualmente dirigen de los Proyectos de Investigación Orientados (PIO), aprobados por resolución 2049/16 del Directorio de CONICET conjuntamente con la Universidad Nacional de Catamarca (UNCa);

establecer las áreas del conocimiento a las cuales pertenecen los perfiles de los doce directores por medio de la información proporcionada por ellos mismos a los fines de su evaluación y publicada en el sistema de Intranet del CONICET;

determinar el nivel de productividad de los doce directores de PIO a partir esencialmente de CONICET Digital e Intranet de cada uno de ellos; y

Entrevistar a los directores, a fin de conocer su percepción sobre los distintos objetivos abordados por este estudio.

Lo expresado previamente se evalúa en un contexto marcado por la incorporación en Argentina de la Convención Interamericana para Prevenir, Sancionar y Erradicar la Violencia contra la Mujer, conocida como Convención de Belém do Pará (sitio de su adopción en 1994). Texto que afirma literalmente "que la violencia contra la mujer constituye una violación de los derechos humanos y las libertades fundamentales y limita total o

acción inicial fue la realización del Primer Censo Científico Técnico, a fin de recopilar información sobre los proyectos de investigación en el ámbito público y privado (LÓPEZ MONRROY, 2014: 30) 
parcialmente a la mujer el reconocimiento, goce y ejercicio de tales derechos y libertades”. Además, dicha violencia "es una ofensa a la dignidad humana y una manifestación de las relaciones de poder históricamente desiguales entre mujeres y hombres".

La ratificación por parte de Argentina del instrumento de la Convención de Belém do Pará fue el 07 de mayo de 1996. Este acto internacional designa el consentimiento del Estado para que un tratado sea obligatorio en su propio territorio [Arts. 2 (1) (b), 14(1) y 16, Convención de Viena sobre el derecho de los tratados de 1969]. De este modo, se obliga a erradicar toda forma de discriminación a la mujer tanto aquella que se produzca en el ámbito público como en el privado.

\section{MATERIAL Y MÉTODOS}

La taxonomía se ha concentrado en las trayectorias académicas y profesionales de las cinco mujeres en comparación con las de los siete varones, a través de la información proporcionada por ellos en el marco de su evaluación y publicada en intranet del CONICET. El análisis parte de los títulos obtenidos, la categoría que reviste cada uno en CIC, la cantidad de tesis de doctorado y maestría dirigidos hasta el momento y los avances en proyectos de investigación previos. Asimismo, a fin de estudiar las diferencias de géneros se han seleccionado y examinado cuatro variables: el número de trabajos publicados, el número de citas obtenidas, número de citas internacionales y el promedio de citas (TORRES, MUÑOZ y JIMENÉZ, 2011: 11)

En el afán de obtener una imagen lo más próxima posible al objeto en estudio se aplicaron las técnicas propias del estudio de caso, análisis de documentos -en particular la resolución 2049/16 del CONICET-, entrevistas semiestructuradas a los doce investigadores e indicadores en Ciencia y Tecnología aplicados a la presencia de la mujer en el Sistema Nacional de Innovación de Argentina.

\section{RESULTADOS}

La primera observación que se puede hacer en relación con este estudio es que ha aumentado significativamente la masa crítica de investigadores en los últimos años, este aumento también significó un impacto en forma positiva en el porcentaje de presencia de mujeres, que se vio incrementado. Sin embargo, el resabio de la exclusión conceptual -discriminación- es que la mayoría femenina se da solo al comienzo de la

\footnotetext{
${ }^{4}$ https://portinos.com/la-mateada/primera-mujer-que-preside-el-conicet-en-50-anos

${ }^{5}$ http://www.oas.org/juridico/spanish/firmas/a-61.html
} 
carrera. El techo de cristal se vuelve visiblemente ostensible en el intento de crecimiento, así se la excluye de la construcción real de decisión.

"En la mayoría de los países del mundo la inserción de las mujeres en el sector científico-tecnológico es un tema crucial, pero no siempre se encuentra en las agendas políticas. Al no considerar este tipo de estudios con una prioridad crítica, existe un inadecuado registro de los datos discriminados por sexo, a pesar del compromiso surgido en la Conferencia Internacional de Beijing (1995), reiterado en la Conferencia Mundial sobre Ciencia celebrada en Budapest2 (1999); donde se trató la necesidad y exigencia de contemplar las variables de sexo y edad en todas las estadísticas sociales, por parte de los organismos responsables" (FRANCHIN, ATRIO, MAFFIA y KOCHEN, 2008: 732)

En este contexto, cobra relevancia la afirmación de una de las principales especialista en Argentina: "la participación diferencial de hombres y mujeres en las actividades de investigación y el análisis de las especificidades en el desarrollo de las carreras científicas femeninas no ha sido objeto de una indagación sistemática ni sostenida en el tiempo. Este déficit en el conocimiento brinda un factor sociológico clave para la comprensión de la estructura científica del país" (ESTÉBANEZ, 2003: 2)

\section{Figura 1: Inserción de la Mujer en el CONICET de Argentina}

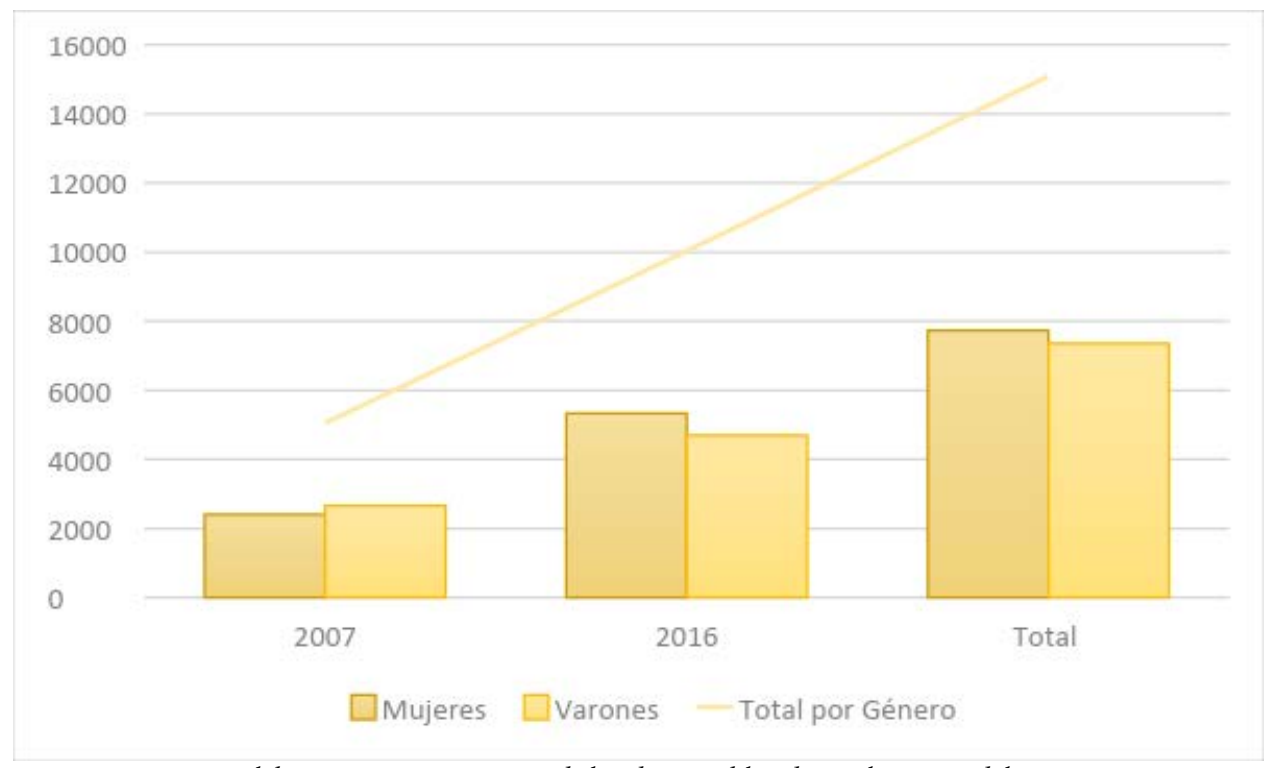

Fuente: Elaboración propia a partir de los datos publicados en la página del CONICET.

"En sus comienzos, el CONICET mantuvo un gran desequilibrio entre los sexos de sus investigadores y entre las diversas especialidades. Mientras que medicina acaparaba un 62\% de varones entre sus investigadores, las Ciencias Sociales y Humanas, donde había mayor cantidad de mujeres, solo ostentaban un 15\% de científicas y esa proporción adquiere otra dimensión cuando se hace un análisis de la ubicación geográfica donde ellas se 
insertaron. De 300 investigadores de carrera solo había 42 mujeres. Para 1965 solo el 9\% de los científicos argentinos era de dicho sexo mientras que en 1971, de un total de 490 investigadores de carrera las mujeres habían aumentado a 72. Eran pocas, pero, si comparamos la situación con otros países latinoamericanos, para fines de los sesenta, la Argentina estaba ingresando al sistema y su participación era cada vez más significativa” (VERA, 2007: 64)

El interesante trabajo elaborado por investigadores en el exterior denominado Women Leaving Science permite evaluar la situación de la mujer de un modo global, así es posible advertir que "la percepción de las mujeres sobre la discriminación sexual y el doble estándar prevaleció entre las entrevistadas". Sin embargo, esa discriminación fuer solo un factores secundario en las decisiones de abandonar la carrera, ya que contribuyeron a la determinación los bajos niveles de tutoría, una falta de coincidencia de intereses y dificultades para asumir la doble carga de la familia y la carrera. Además, muchas de estas mujeres habían convivido con la discriminación sexual percibida desde la escuela secundaria y habían encontrado estrategias para persistir en la ciencia a pesar del trato desigual (PRESTON, 2006: 13)

Asimismo, "las mujeres, más que los varones, estaban insatisfechos con que en esencia el trabajo no se conectaba con los problemas de la vida real que consideraban importantes" (PRESTON, 2006: 14)

El CONICET tiene una carrera del investigador con una jerarquía establecida por Ley 20464/73 en cinco niveles, de menor a mayor son las siguientes con su respectiva delimitación de aptitudes: Asistente (labor personal de investigación científica, o algún desarrollo o labor tecnológica creativos, así como poseer la preparación técnica necesaria para desarrollar un tema por sí mismo), Adjunto (capacidad de planear y ejecutar una investigación o desarrollo, así como de colaborar eficientemente en equipos), Independiente (trabajos originales de importancia en investigación científica o en desarrollo, además de estar en condiciones de elegir los temas, y planear y efectuar las investigaciones en forma independiente, o haberse distinguido como miembro de un equipo de reconocida competencia), Principal (amplia labor científica o de desarrollo tecnológico, de originalidad y alta jerarquía reconocida, revelada por sus publicaciones y por la influencia de sus trabajos en el adelanto de su especialidad en el campo de la ciencia o de la técnica) y, finalmente, Superior (extensa labor original de investigación científica o de desarrollo tecnológico, de alta jerarquía que lo sitúe entre el núcleo de los especialistas reconocidos en el ámbito internacional). La promoción de una categoría a la siguiente se obtiene luego de una rigurosa evaluación sobre el desempeño realizada por comisiones de pares, que observan especialmente sus obras, según criterios cualitativos (subsidiariamente cuantitativos), así como su capacidad para transmitir conocimientos y experiencias directamente en relación con su actividad como investigador, dirigir y formar personal de investigación. En las clases Principal y Superior se tiene especialmente en consideración la 
actividad llevada a cabo en la creación, organización y/o desarrollo de centros de investigación o su actuación en organismos de planeamiento, promoción o ejecución científica.

\section{Figura 2: Permanencia y Promoción de la Mujer en el CONICET de Argentina}

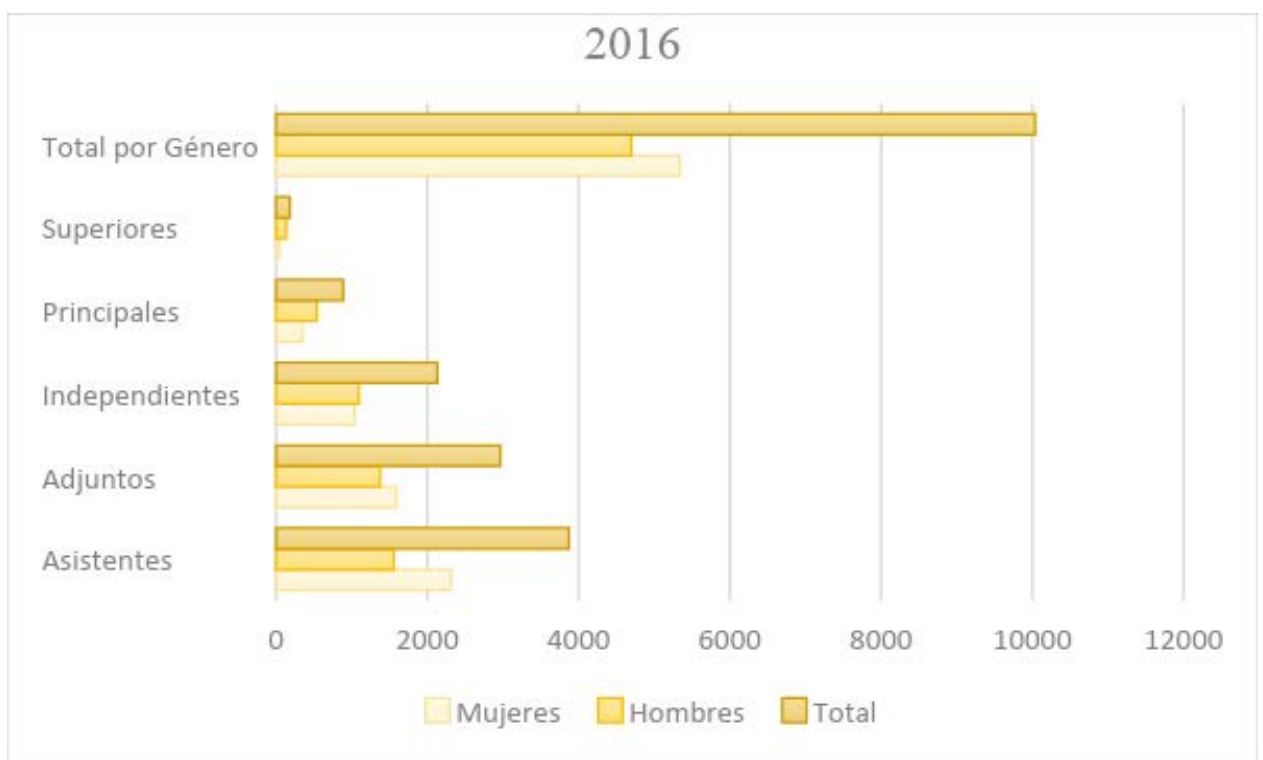

Fuente: Elaboración propia a partir de datos publicados en la página del CONICET.

El extremo de la inequitativa distribución por género lo constituye precisamente la muestra representativa del estudio, esto es, el Centro de Investigación y Transferencia de Catamarca (CITCA), así en el inicio de la carrera hay veintiún (21) becarias, pero en las categorías Principal y Superior existe solo un varón en cada una de ellas, tal como podrá observarse en la figura que sigue a continuación:

\section{Figura 3: Distribución de la Mujer por Categoría en el CITCA}

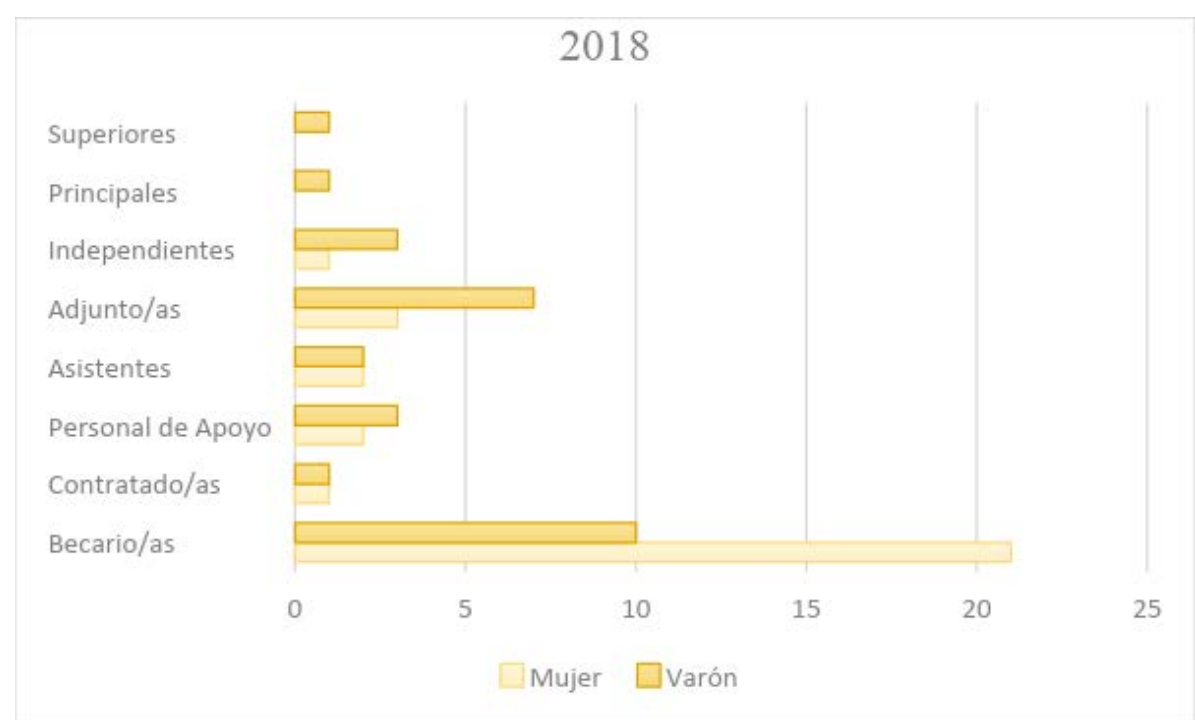

Fuente: Elaboración propia a partir de datos publicados en la página del CONICET. 
En este sentido, el fenómeno que se puede advertir es que existe una dificultad más fuerte para crecer que el nivel deserción en Argentina, a diferencia de otros países como Estados Unidos en el que el abandono de la carrera es mayor. Durante el período de 1993 a 1999, el 17.6 por ciento de varones dejó las ciencias naturales mientras ese porcentaje en las mujeres se ha incrementado a un 29.1 por ciento. Esos porcentajes en las ciencias sociales son de 12.8 de los varones y el 15.5 de las mujeres para el mismo período (PRESTON, 2006: 7-8)

"En la posición diferencial de los sexos en la ciencia centroamericana y algunas tendencias históricas, los fenómenos más notables de disparidad se localizan en el acceso a puestos de prestigio científico, en las instancias de reconocimiento social y en el nivel de toma de decisiones" (ESTÉBANEZ y LÁSCARIS COMNENO, 2004: 8)

En 1994, el informe elaborado desde la Red Argentina de Género, Ciencia y Tecnología surgió de la necesidad de visibilizar la situación específica de las mujeres en un periodo crítico de la política científica y tecnológica nacional y regional, constatada la ausencia de datos provistos desde el Estado para ese diagnóstico ( $\mathrm{y}$, por tanto, para políticas públicas especialmente dirigidas a las mujeres en estas áreas) y de un compromiso con el logro de situaciones laborales equitativas. Uno de los objetivos de la creación fue trazar un diagnóstico de la situación de la mujer en ciencia y tecnología en distintas áreas del conocimiento y su evolución en los últimos años para poder elaborar estrategias de promoción y valorización de la contribución de las mujeres en ciencia y tecnología. Los primeros resultados encontrados dieron cuenta de la situación de la mujer que sugería que su ausencia en los lugares significativos no era proporcional a la evolución de la matrícula universitaria. En entrevistas realizadas a becarias, investigadoras jóvenes e investigadoras formadas pudieron detectarse algunas formas sistemáticas de barreras relacionadas al sexo, como disminución en el monto de las becas, no contemplación de licencia y otros derechos relacionados con la maternidad, no existencia de becas externas de corta duración, disparidad en la distribución de jerarquías con los mismos antecedentes y hostigamientos en el ambiente de trabajo. ${ }^{6}$

Ahora bien, en relación con el segundo objetivo, esto es, "comparar las trayectorias académicas y profesionales de las cinco mujeres en relación con las de los siete varones, que actualmente dirigen de los Proyectos de Investigación Orientados (PIO), aprobados por resolución 2049/16 del Directorio de CONICET conjuntamente con la Universidad Nacional de Catamarca (UNCa)". En este sentido, el título de los PIO dirigidos por una mujer son los que siguen a continuación:

Estudios experimentales y teóricos de materiales nanoestructurados para su aplicación en electrocatálisis

\footnotetext{
${ }^{6}$ Red Argentina de Género, Ciencia y Tecnología (RAGCyT)
} 
y biosensores;

Asociación de nuevos materiales nanoestructurados con tecnologías verdes para el control del proceso y mejora de la calidad del aceite de oliva;

Diseño de líneas de base e indicadores biológicos para monitoreo ambiental de ecosistemas microbianos asociados a minerales en zonas de interés minero en Catamarca. Estudios de Resiliencia;

Aislamiento e identificación de hongos filamentosos de la micodiversidad de Catamarca, Argentina; con capacidad detoxificante de residuos oleícolas provenientes de la industria local; y

Rescate, descripción y puesta en valor del patrimonio histórico documental de Catamarca y su transferencia para la construcción del conocimiento socio histórico.

Mientras que los títulos de los PIO dirigidos por un varón se describen a subsiguientemente:

Sistema de conversión y almacenamiento de energía para vehículos eléctricos con celdas de combustible de hidrogeno y baterías de ion litio;

Bioprospección del ambiente marino subantártico, en búsqueda de extremocinas para su uso en control biológico en la industria olivícola. Derivación de andrimida por biotransformación;

Generación Eléctrica Solar Dish Stirling;

Territorios y cuerpos en el Siglo XXI. Transformaciones socioterritoriales en la Provincia de Catamarca entre 1990 y 2015. Problemáticas y perspectivas frente a los desafíos del desarrollo, la democratización y la sustentabilidad;

Modos de vida agropastoriles en ambientes contrastantes. Casa y territorio en las Serranías de El Alto Ancasti y Antofagasta de la Sierra, Catamarca. Primer y segundo milenios D.C.;

Manejo Integrado de los principales insectos plaga de la fruticultura en la Provincia de Catamarca; y

Impacto de las políticas socioeducativas de inclusión social en trayectorias biográficas y sociales precarizadas de jóvenes catamarqueñ@s.

El primer dato que surge de la información que antecede es que más cantidad de proyectos dirigidos por varones (7) que mujeres (5). Por ello, resulta interesante conocer cuál es la categoría que reviste cada una/o de los ellas/os, así en relación con la posición jerárquica al interior de CONICET es relevante destacar en este punto que el ascenso se produce solo por recomendación de pares. Los resultados de las mujeres son los que siguen a continuación:

1. Investigadora independiente;

2. Investigadora independiente;

3. Investigadora principal; 
4. Investigadora independiente; $\mathrm{e}$

5. Investigadora principal.

6. En este contexto, las categorías de los varones son las siguientes:

7. Investigador asistente al momento de la resolución 2049 en mayo de 2016, antecedente que le fue útil para lograr su promoción a la categoría de Adjunto en diciembre de ese mismo año;

8. Investigador independiente;

9. Investigador principal;

10. Investigador adjunto;

11. Investigador adjunto;

12. Investigador adjunto;

13. Investigador independiente al momento de la mencionada resolución también promovido, en este supuesto a principal, en diciembre 2016.

La dirección de un proyecto de la envergadura de los de investigación orientada requiere, según la reglamentación vigente que la persona debe poseer la categoría de investigador/a independiente. Sin embargo, cuatro de los siete varones no revisten esa categoría.

He aquí la diferencia sustancial con las mujeres, pues todas ellas poseen la categoría de independiente o, incluso, una superior a ella al momento de presentación a la convocatoria. Dato que enorgullece a las mujeres que hacen ciencia, ya que a menudo se confunde y se cree que se espera un trato diferencial por el solo hecho de ser mujer, pero lo que se reclama es simplemente la consideración del mérito cuando existe, esto es, que idénticas trayectorias sean merituadas de la misma manera se sea mujer o varón; al igual que lo que sucede con la participación de la mujer en otros ámbitos laborales del país.

El análisis de la muestra en virtud de los títulos académicos alcanzados por unas y otros se esquematiza en el mismo orden de los PIO, primero las mujeres y luego los varones, del siguiente modo:

1. Doctora en química y bioquímica en el grado;

2. Doctora en bioquímica y bioquímica de título de grado;

3. Doctora en biología y licenciada en biología;

4. Posdoctora en reología y modelado matemático, doctora en bioquímica y bioquímica en el grado; y

5. Doctora en historia y licenciada también en historia.

6. En el caso de los varones, los datos en relación con el mismo parámetro son los siguientes:

7. Doctor en energía e ingeniero mecánico electricista;

8. Posdoctor del CONICET, doctor de la Universidad Nacional de Tucumán con orientación genética y 
licenciado en genética;

9. Doctor en física y licenciado en física;

10. Magister en ciencias sociales con mención en teoría política, especialista en planificación social para el desarrollo local y licenciado en ciencia política, pero no carece de título de doctor;

11. Doctor en ciencias naturales y licenciado en arqueología de título de grado;

12. Doctor en ciencias biológicas, magister en entomología aplicada y licenciado en ciencias biológicas con orientación zoología; y

13. Doctor en filosofía y ciencias de la educación, magister en metodología de la investigación científica, especialista en psicogerontología, especialista en metodología de la investigación científica, licenciado en ciencias de la educación y profesor de ciencias jurídicas, políticas y sociales.

Nuevamente, se observa que mientras que todas las mujeres ostentan el título de doctor que demuestra que han sido capaces de llevar adelante una investigación, esto es, al menos la propia; no todos los varones detentan ese mérito. Más aún, cuando la exigencia de dicho título es condición indispensable, o al menos así lo sostiene la normativa, para el ingreso a la carrera de investigador del CONICET.

El tercer objetivo de la presente investigación consiste en "establecer las áreas del conocimiento a las cuales pertenecen los perfiles de los doce directores por medio de la información proporcionada por ellos mismos a los fines de su evaluación y publicada en el Sistema de Intranet del CONICET". A simple vista parece existir diversidad disciplinar. Por ello, se ha profundizado en el área académica de procedencia de cada una de las investigadoras y sus resultados son:

1. Química-Física;

2. Bioquímica;

3. Biología;

4. Biotecnología; $y$

5. Ciencias Sociales y Humanas.

El primer, segundo, tercero y cuarto proyecto dirigido por una mujer pertenecen a las áreas consideradas exactas "o duras" como también frecuentemente se las denomina. Solo la quinta de las propuestas es del área de las ciencias sociales y humanas, en particular de la historia. En consecuencia, es posible advertir que esa distinción que aún se cierne en el inconsciente colectivo de que hay ramas del conocimiento reservadas en exclusividad a la mujer no se da en esta investigación.

"Antes era un lugar común escuchar afirmar, escuetamente, a científicos, maestros y padres que las mujeres no pueden, ni deben, ser científicas, que les falta la fuerza, el rigor y la claridad de mente necesarias para 
una ocupación que pertenece a los hombres. Ahora, cuando el movimiento de mujeres ha hecho que resulten ofensivas afirmaciones tan desnudas, el reconocimiento abierto de la creencia, que sigue estando en vigor, acerca de la masculinidad intrínseca del pensamiento científico esta menos de moda. Y, sin embargo, se sigue encontrando la expresión diaria en el lenguaje y las metáforas que usamos para describir la ciencia. Cuando apodamos duras a las ciencias objetivas en tanto que opuestas a las ramas del conocimiento más blandas (es decir, más subjetivas), implícitamente estamos invocando una metáfora sexual en la que por supuesto dura es masculino y blanda es femenino. De forma general, los hechos son duros, los sentimientos blandos. Feminización se ha convertido en sinónimo de sentimentalización. Una mujer que piensa científica u objetivamente está pensando como un hombre; a la inversa, el hombre que siga un razonamiento no racional, no científico, está argumentando como una mujer" (FOX KELLER, 2001: 159)

Las mujeres quedan así excluidas del saber científico en tanto se identifican con el lugar de la emoción, los afectos y la intuición. La noción tradicional de objetividad presenta a la ciencia como una actividad hostil para las mujeres, pues sus métodos y definiciones entran en colisión con la construcción social de femineidad (FERNÁNDEZ RIUS, 2010:4)

En idéntico sentido, en el caso de los proyectos dirigidos por un varón corresponden a las siguientes áreas del conocimiento:

1. Desarrollo Tecnológico y Social;

2. Genética;

3. Física;

4. Ciencias Sociales y Humanas;

5. Ciencias Sociales y Humanas;

6. Biología; $y$

7. Ciencias Sociales y Humanas.

El primero de los proyectos procede del desarrollo tecnológico y social proyectos complejos, en cambio el segundo pertenece a la genética. El tercero corresponde a la física, en especial a la física teórica. El cuarto, se sitúa en los estudios sociales o humanos. El quinto también pertenece a las ciencias sociales, pero más precisamente al área de la arqueología y preservación del patrimonio. El sexto se ubica en el sector de la biología aplicada a plagas. Finalmente, el séptimo aborda la producción de conocimientos propios de las ciencias sociales y humanas.

La ciencia y la tecnología no son neutrales, sino que, al igual que todos los demás modos de ordenar la realidad y de entender los datos, nacen en un contexto social e histórico que tiene unos valores y unos intereses sociales intrínsecos en sus estructuras. Si bien estos contextos han ido cambiando, hay un rasgo que ha 
permanecido inalterado desde el origen mismo de las ciencias: su carácter androcéntrico. Los hombres han participado exclusivamente en su construcción y en decidir los intereses de la misma. El cuestionamiento acerca de quién hace la ciencia y para quién ha planteado la posibilidad de que la incorporación de la mujer (entre los diferentes actores ausentes en la construcción del conocimiento) resultaría un aporte positivo para socializar los objetivos del conocimiento científico (PÉREZ SELDEÑO, 2001:22)

El cuarto objetivo, por su parte, intenta "determinar el nivel de productividad de los doce directores de PIO”. Para ello, la fuente fundamental de información en este sentido es el repositorio CONICET Digital, cuya administración está a cargo de un grupo de bibliotecarios especializados que conforman la Oficina Técnica Central de tal unidad de información.

Toda la producción científico-técnica autoarchivada en SIGEVA CONICET no se incorpora automáticamente en CONICET Digital. El pasaje desde SIGEVA CONICET hacia el Repositorio Institucional CONICET Digital, no es automático dado que antes de ser publicada una producción pasará por la Oficina Técnica donde bibliotecarios y especialistas, realizan el proceso de curatoría de datos, es decir, se rectifican los datos bajo normas, directrices y estándares internacionales y revisan su disponibilidad en cuanto a las políticas editoriales existentes.

Además, si la producción ha sido autoarchivada por varios coautores, también media un proceso de compilación automática para reconocer los registros semejantes (autoarchivados por varios coautores) y preparar los lotes de registros que pasarán a la etapa de unificación de registros. El siguiente paso consiste en el proceso de unificación que se realiza desde la Oficina Técnica y en el que se revisa cada registro compilado en la etapa anterior, y de forma exhaustiva, se comparan las semejanzas y se selecciona de cada registro la descripción del metadato mejor elaborado. De esta manera, de cada producción habrá un solo registro con todos los autores y filiaciones de los mismos que han intervenido en ella.

La primera mujer de las directoras de los PIO tiene siete publicaciones en colaboración actualmente en CONICET Digital cuya revista, editorial, idioma y año son los siguientes:

1. Aportes Científicos en PHYMATH (Universidad Nacional de Catamarca. Facultad de Ciencias Exactas y Naturales), Español en 2013;

2. Applied Surface Science (Elsevier Science), Inglés en 2017;

3. Journal of Electroanalytical Chemistry (Elsevier Science), Inglés en 2017;

4. Electrochemistry Communications (Elsevier Science), Inglés en 2013;

5. IEEE Transactions on Magnetics (Institute of Electrical and Electronics Engineers), Inglés en 2013;

6. Faraday Discussions (Royal Society of Chemistry), Inglés en 2014; y 
7. Electrochimica Acta (Pergamon-Elsevier Science Ltd.), Inglés en 2014.

La producción adicional de la investigadora en su Intranet consta de un total de treinta y tres (33) publicaciones en inglés y español prioritariamente a través de la editorial Elsevier Science.

La segunda mujer de las directoras de los PIO posee cinco publicaciones colaborativas en CONICET Digital cuya revista, editorial, idioma y año son los siguen a continuación:

1. Progress in Neurobiology (Pergamon-Elsevier Science Ltd.), Inglés en 2018;

2. Biochimie (Elsevier Science), Inglés en 2013;

3. Journal of Biological Chemistry (American Society for Biochemistry and Molecular Biology), Inglés en 2014;

4. Journal of Biological Chemistry (American Society for Biochemistry and Molecular Biology), Inglés en 2014;

5. RSC Advances (Royal Society of Chemistry), Inglés en 2016.

Las publicaciones de la investigadora en su Intranet CONICET son veintinueve (29) publicaciones en inglés y, especialmente, a través de las editoriales Naturey Elsevier Science.

La tercera directora posee una realmente abundante producción colaborativa -en todos los sentidos- en CONICET Digital, que suma un total de cuarenta y nueve (49) publicaciones. Sus datos son los siguientes:

1. Microbial Ecology (Springer), Inglés en 2015;

2. Revista Hipótesis (Universidad de Los Andes), Español en 2012;

3. Extremophiles (Springer Tokyo), Inglés en 2014;

4. Genome Announcements (American Society for Microbiology), Inglés en 2013;

5. Genome Announcements (American Society for Microbiology), Inglés en 2015;

6. Reduca (Universidad Complutense de Madrid), Español en 2014;

7. Current Microbiology (Springer), Inglés en 2006;

8. Genome Announcements (American Society for Microbiology), Inglés en 2013, Volume 1 Issue 4 e00480-13;

9. Genome Announcements (American Society for Microbiology), Inglés en 2014;

10. FEMS Microbiology Letters (Elsevier Science), Inglés en 2000;

11. Applied Microbiology and Biotechnology (Springer-Verlag Berlin), Inglés en 2012;

12. Microbial Ecology (Springer), Inglés en 2009;

13. Photochemical and Photobiological Sciences (Royal Society of Chemistry), Inglés en 2104;

14. Plasmid (Academic Press Inc Elsevier Science), Inglés en 2010; 
15. Photochemistry and Photobiology (Wiley Blackwell Publishing, Inc.), Inglés en 2016;

16. Plos One (Public Library of Science), Inglés en 2016;

17. BMC Genomics (BioMed Central), Inglés en 2014;

18. Frontiers in Microbiology (Frontiers Editorial Office-EPFL), Inglés en 2015;

19. International Journal Of Systematic And Evolutionary Microbiology (Society For General Microbiology), Inglés en 2011;

20. Extremophiles (Springer Tokyo), Inglés en 2016;

21. Frontiers in Microbiology (Frontiers Research Foundation), Inglés en 2015;

22. Hydrobiologia (Springer), Inglés en 2006;

23. Recent Patents on Anti-Infective Drug Discovery (Bentham Science Publishers), Inglés en 2009;

24. Journal of General and Applied Microbiology (Microbiology Research Foundation), Inglés en 2009;

25. Plos One (Public Library of Science), Inglés en 2016;

26. Extremophiles (Springer), Inglés en 2013;

27. Isme Journal (Nature Publishing Group), Inglés en 2015;

28. Isme Journal (Nature Publishing Group), Inglés en 2016;

29. Microbial Ecology (Springer), Inglés en 2014;

30. Frontiers in Microbiology (Frontiers Editorial), Inglés en 2016;

31. Plos One (Public Library of Science), Inglés en 2016 (p:1-21);

32. Journal of Basic Microbiology (Wiley VCH Verlag), Inglés en 2010;

33. Current Microbiology (Springer), Inglés en 2008;

34. Journal Of South American Earth Sciences (Elsevier Science), Inglés en 2013;

35. Revista Latino Americana de Microbiología (Asociación Mexicana de Microbiología), Inglés en 2004;

36. Plos One (Public Library of Science), Inglés en 2017;

37. Extremophiles (Springer Tokyo), Inglés en 2013;

38. Applied And Environmental Microbiology (American Society for Microbiology), Inglés en 2008;

39. Biotechnology Letters (Springer), Inglés en 2001;

40. Frontiers in Microbiology (Frontiers Editorial), Inglés en 2015;

41. Frontiers in Microbiology (Frontiers Editorial), Inglés en 2017;

42. Biotechnology Letters (Springer), Inglés en 2010;

43. Applied Cell Biology (Trade Science), Inglés en 2015;

44. Plos One (Public Library of Science), Inglés en 2013; 
45. Genome Announcements (American Society for Microbiology), Inglés en 2014, Volume 1 Issue 6 e00885-13;

46. PeerJ (PeerJ Inc.), Inglés en 2017;

47. Extremophiles (Springer Tokyo), Inglés en 2014;

48. Journal of Photochemistry and Photobiology B: Biology (Elsevier Science), Inglés en 2013; y

49. FEMS Microbiology Letters (Wiley Blackwell Publishing, Inc.), Inglés en 2011.

50. Las publicaciones de esta autora ascienden a un total de ciento veintiséis (126) en Intranet CONICET. La mayoría en inglés y por medio de las editoriales Naturey Elsevier Science.

51. La cuarta directora de los PIO del CITCA tiene diecinueve (19) publicaciones en colaboración validadas por CONICET Digital. Los detalles de dichos artículos en la misma línea de sus predecesoras son:

52. Enzyme and Microbial Technology (Elsevier Science), Inglés en 2002;

53. Food Hydrocolloids (Elsevier Science), Inglés en 2006;

54. Research Journal of Microbiology (Academic Journals), Inglés en 2010;

55. International Biodeterioration and Biodegradation (Elsevier Science), Inglés en 2014;

56. Acta Biológica Colombiana (Universidad Nacional de Colombia), Inglés en 2015;

57. Water, Air and Soil Pollution (Springer), Inglés en 2010;

58. Enzyme and Microbial Technology (Elsevier Science), Inglés en 2007;

59. World Journal of Microbiology (Springer), Inglés en 2014;

60. Carbohydrate Polymers (Elsevier Science), Inglés en 2013;

61. International Biodeterioration And Biodegradation (Elsevier Science), Inglés en 2011;

62. Frontiers in Microbiology (Frontiers Editorial), Inglés en 2015;

63. Enzyme and Microbial Technology (Elsevier Science), Inglés en 2008;

64. Antonie van Leeuwenhoek (Springer), Inglés en 2008;

65. Scientific World Journal (Hindawi Publishing Corporation), Inglés en 2012;

66. International Journal of Biological Macromolecules (Elsevier Science), Inglés en 2007;

67. Journal of Applied Microbiology (Wiley Blackwell Publishing, Inc.), Inglés en 2009;

68. Revista Iberoamericana de Micología (Asociación Española Micología), Inglés en 2009;

69. International Biodeterioration And Biodegradation (Elsevier Science), Inglés en 2013; y

70. Anton Leeuw Int. J. G. (Springer), Inglés en 2011.

Mientras que sus publicaciones en Intranet CONICET son treinta y cuatro (34). Prioritariamente en 
inglés, a través de Elsevier Science.

Ahora bien, la única excepción al notable aporte al conocimiento de las directoras lo constituye la quinta de ellas, pues no ostenta ninguna publicación que haya superado la evaluación del equipo de bibliotecarios del CONICET. En su Intranet figuran quince (15) artículos en revistas nacionales e idioma español. Tres de ellas en colaboración y doce de su exclusiva e individual autoría.

Por su parte, el primer varón de los directores posee solo tres (3) producciones en CONICET Digital. Todas las publicaciones lo tienen como primer autor y se trata de trabajos en colaboración. Ellas son las que siguen a continuación:

1. Revista de la Facultad de Ciencias Exactas Físicas y Naturales (Universidad Nacional de Córdoba. Facultad de Ciencias Exactas, Físicas y Naturales), Español en 2014;

2. Proceedings of the Institution of Mechanical Engineers Part G-Journal of Aerospace Engineering (Professional Engineering Publishing Ltd.), Inglés en 2015; y

3. International Journal of Hydrogen Energy (Pergamon-Elsevier Science Ltd.), Inglés en 2015.

En Intranet CONICET se mencionan trece (13) artículos de su autoría en colaboración con otros investigadores.

El segundo director exhibe la valiosa labor de catorce (14) artículos agregados a CONICET Digital. Las referencias de estas producciones en colaboración son:

1. Enzyme and Microbial Technology (Elsevier Science), Inglés en 2005;

2. World Journal of Microbiology \& Biotechnology (Springer), Inglés en 2013;

3. Research Journal of Microbiology (Academic Journals), Inglés en 2010;

4. Antonie van Leeuwenhoek (Springer), Inglés en 2004;

5. International Journal of Systematic and Evolutionary Microbiology (Society for General Microbiology), Inglés en 2004;

6. Extremophiles (Springer Tokyo), Inglés en 2008;

7. Carbohydrate Polymers (Elsevier Science), Inglés en 2013;

8. International Journal of Systematic and Evolutionary Microbiology (Society for General Microbiology), Inglés en 2004;

9. World Journal of Microbiology (Springer), Inglés en 2006;

10. Biotechnology Letters (Springer), Inglés en 2004;

11. International Journal of Systematic and Evolutionary Microbiology (Society for General Microbiology), Inglés en 2015; 
12. Biotechnology Letters (Springer), Inglés en 2005;

13. International Biodeterioration And Biodegradation (Elsevier Science), Inglés en 2013; y

14. Anton Leeuw Int. J. G. (Springer), Inglés en 2011.

En Intranet CONICET se mencionan treinta y nueve (39) artículos en colaboración. Todos ellos en inglés y publicados en revistas internacionales.

El tercer director posee solo dos (2) artículos en CONICET Digital. Uno de ellos como primer autor y el otro como parte del equipo de investigación. Su detalle es el siguiente:

1. Nature Photonics (Nature Publishing Group), Inglés en 2013; y

2. Physical Review D (Amer Physical Soc.), Inglés en 2013.

La Intranet CONICET tiene registrados veinticinco (25) publicaciones en colaboración de este director. Todas ellas en inglés y divulgadas en revistas internacionales.

El cuarto director ha publicado un solo artículo validado por CONICET Digital. Este único artículo es en español y labor individual.

Onteaiken (Centro de Investigaciones y Estudio sobre Cultura y Sociedad), Español en 2013.

Advierta el lector que este director es quien, además, no posee título de doctor. La afirmación indica que para su ingreso a CIC tampoco fue por el peso de sus publicaciones. Por ello, no hay ningún mérito que sustente su ingreso o permanencia en el sistema.

Este mismo director tiene treinta y ocho (38) publicaciones agregadas en su Intranet de CONICET.

El quinto director también posee un solo artículo como coautor y en español certificado por CONICET Digital. Sus datos son:

Revista Española de Antropología Americana (Universidad Complutense de Madrid), Español en 2013.

Los datos incorporados por este director en su Intranet de CONICET corresponden a treinta y un (31) artículos en español e inglés.

El sexto director presenta catorce (14) publicaciones validadas por CONICET Digital, cuyos detalles se mencionan a continuación:

1. Biological Control (Elsevier Science), Inglés en 2016;

2. Natural Science (Scientific Research), Inglés en 2014, 6, 664-675;

3. Insects (MDPI), Inglés en 2012;

4. Biocontrol Science And Technology (Taylor \& Francis Ltd.), Inglés en 2013;

5. Florida Entomologist (Florida Entomological Society), Inglés en 2010;

6. Florida Entomologist (Florida Entomological Society), Inglés en 2004; 
7. Biological Invasions (Springer), Inglés en 2014;

8. Biological Control (Elsevier Science), Inglés en 2004;

9. Ecological Entomology (Wiley-blackwell Publishing, Inc.), Inglés en 2013;

10. Journal of Insect Behaviour (Springer), Inglés en 2017;

11. Natural Science (Scientific Research), Inglés en 2014, 6, 1267-1274;

12. Florida Entomologist (Florida Entomological Society), Inglés en 2008;

13. Florida Entomologist (Florida Entomological Society), Inglés en 2006; y

14. Florida Entomologist (Florida Entomological Society), Inglés en 2005.

Los antecedentes asociados por este director en su Intranet de CONICET pertenecen a treinta y dos (32) artículos en inglés publicados en revistas internacionales.

El séptimo y último director detenta solo tres (3) obras en CONICET Digital. Dos de ellas en español y una en inglés. Los tres artículos son trabajos colectivos y sus detalles son los siguientes:

1. Publicatio (Universidade Estadual de Ponta Grossa), Español en 2013;

2. Revista de Docencia Universitaria (Red Estatal de Docencia Universitaria), Español en 2014; y

3. Educational Gerontology (Taylor \& Francis), Inglés en 2014.

Corroborada la hipótesis derivada de que son las mujeres quienes reúnen sobradamente todas las exigencias, así es posible observar la vasta producción de ellas en un contexto marcado por la profundidad y diversidad de temas, destinos e idiomas de sus trabajos.

En este contexto, "a nivel internacional encontramos el análisis del Council of Scientific and Industrial Research (CSIR) de Nueva Delhi que concluye que no existen diferencias en la productividad de los científicos masculinos y femeninos (Gupta y otros, 1999), conclusiones similares a las que se alcanzan en estudios posteriores (Palomba y Menniti, 2001; Lewison, 2001). Sin embargo distintos estudios sobre el profesorado universitario realizados en otros países como Italia o Croacia si identifican contrastes significativos en la productividad de hombres y mujeres. En el caso italiano son mejores los resultados de los hombres, aunque existen determinados sectores científicos en los que las mujeres se muestran más productivas (Abramo y otros, 2009); en el croata incluso las mujeres más productivas publican menos que sus homólogos masculinos (Prpic, 2002)" (TORRES ET AL, 2011: 13)

El abordaje del quinto y último objetivo de este trabajo, esto es, "entrevistar a los directores, a fin de conocer su percepción sobre los distintos objetivos abordados por este estudio". La idea original en relación a este objetivo era una entrevista personal semiestructurada con cada uno de los directores, sin embargo ante la solicitud de una de las directoras la entrevista se les envió un cuestionario por e-mail. Luego del envío, la primera en 
responder inmediatamente fue una directora mujer y solicito que la entrevista se hiciese por teléfono, hecho que enriqueció mucho el intercambio.

La sorpresa del objetivo planteado vino de la mano de que solo tres de las cinco directoras mujeres han sido quienes respondieron a las preguntas. Dos de ellas en forma inmediata y la restante contestó a los once días de la recepción del texto. Mientras que en el caso de los varones uno respondió a los diez días, pero indicando que no tenía tiempo para realizar la entrevista en forma escrita. Idéntica respuesta se obtuvo de otro director varón, sin embargo esa respuesta se produjo luego de casi un mes (27 días después)

Las preguntas enviadas a los directores de los PIO del CITCA (CONICET/UNCa) y las respuestas de las tres directoras mujeres son las siguientes:

¿Cuáles son los objetivos y características de los Proyectos de Investigación Orientados (PIO) del

\section{CONICET?}

"El objetivo es fortalecer el sistema científico en áreas de vacancia nacional que a su vez estén orientados a temáticas de interés o necesidad regional, propia del lugar donde son implementados, o que respondan a intereses, potencialidades, requerimientos, o inquietudes locales", Directora Mujer 1.

"La iniciativa es buena en relación con el impacto local por ser orientados, sin embargo las dificultades en la asignación de los recursos hace que muchas veces esos montos no alcancen para financiar realmente la actividad. Por ello, sería interesante que se otorgue el monto completo una vez aprobado el proyecto o se actualicen cuando se abonan con una diferencia significativa de tiempo, pues muchas veces se esperan dos o más años para recibir ese importe original", Directora Mujer 2.

"En mi caso particular, los objetivos y características del PIO se enmarcan dentro de proyectos anteriores, en los cuales he sido investigador responsable. Si bien los montos asignados al proyecto ya fueron ejecutados, los objetivos fundamentales se siguen desarrollando. Dentro del proyecto anterior, se comenzó con la formación académica de recursos humanos y la compra de equipamiento. Luego, CONICET inicia una activa vinculación con la UNCa, la cual culmina con la formación de la Unidad Ejecutora. Los objetivos y características de los Proyectos de Investigación Orientados (PIO) del CONICET tratan de reforzar las actividades relacionadas con la profundización del conocimiento en determinadas áreas, las cuales son impulsadas y organizadas por el Directorio del CITCA", Directora Mujer 3.

¿Qué implicancias tienen para Catamarca?

"Son relevantes porque pueden permitir idear soluciones a problemáticas importantes para la provincia, basadas en el conocimiento científico y la I+D, porque fortalecen el sistema de adquisición y apropiación de conocimiento, y además, podrían resultar una fuente de innovación”, Directora Mujer 1. 
"La importancia local radica en el hecho de ser orientados a necesidades puntuales del lugar en el que se insertan”, Directora Mujer 2.

"Creo que el PIO, con el consenso de todos sus integrantes, puede tener grandes implicancias en el progreso de los laboratorios de investigación orientados a la microscopia. Recientemente, se ha adquirido el equipamiento del laboratorio especializado en técnicas electroquímicas para el estudio básico de reacciones electrocatalíticas de vital importancia en celdas de combustible y celdas electrolíticas”, Directora Mujer 3.

¿Qué aptitudes debe tener un investigador para dirigir un PIO?

"Debe tener una sólida y comprobable formación académica y científica, con capacidad para la formación de RRHH, capaz de generar ideas innovadoras y/o vinculantes al sistema productivo, con pensamiento crítico, y estar actualizado sobre las necesidades locales que puedan nutrirse del conocimiento científico", Directora Mujer 1.

"Independencia y experiencia en el contacto con los problemas concretos de la sociedad. No solo a través de publicaciones sino, además, una mirada global al conocimiento e impacto de la experiencia del investigador en la dirección de proyectos anteriores y becarios en función de su eficiencia. Esa eficiencia contribuiría significativamente a asignar más dinero a determinados proyectos con un alto índice de posibilidades de éxito", Directora Mujer 2.

"Las aptitudes que debe tener un investigador para dirigir un PIO, son las mismas que las necesarias para dirigir cualquier otro proyecto", Directora Mujer 3.

¿Usted cree qué la categoría reconocida por pares es relevante al momento de dirigir un proyecto de la envergadura de un PIO?

"Sí, Directora Mujer 1.

"Autoridad para el éxito del proyecto, avalada tanto en la dirección de tesis, becarios y proyectos anteriores. El énfasis debe estar puesto en la contribución efectiva al conocimiento del investigador, esto es, la apertura de líneas de investigación propias", Directora Mujer 2.

"No tengo opinión formada al respecto", Directora Mujer 3.

¿Cuál es la categoría de la carrera de investigador que avala esas aptitudes?

"Desde Independiente", Directora Mujer 1.

La respuesta de la Directora Mujer 2 a esta pregunta se encuentra incluida en su respuesta a la pregunta anterior.

"No tengo opinión formada al respecto", Directora Mujer 3.

¿Usted considera que la mujer tiene aptitudes distintas a los varones? ¿Cuáles serían esas diferencias? 
"A mi parecer, tienen iguales aptitudes. En general tienen un desempeño similar. Solo que a las mujeres muchas veces les implica mayor esfuerzo personal. Creo que si una investigadora no rinde en su cargo es más que nada por cuestiones inherentes a su forma de ser o capacidad, que por una cuestión de género. Sería interesante por ejemplo, al analizar estadísticas, considerar la producción y algún coeficiente que contemple estado civil y/o cantidad de hijos", Directora Mujer 1.

"En mi experiencia, el desempeño de la mujer y el varón es similar o no y en qué actividad puntual. Hay mujeres muy eficientes, como así también hay varones, pues el género en mi experiencia no hace la diferencia”, Directora Mujer 2.

"Considero que la mujer tiene las mismas aptitudes que los varones", Directora Mujer 3.

Actualmente, ¿la obtención del título de doctor es un requisito indispensable para ingresar a Carrera de Investigador de CONICET? Desde qué año es un requerimiento central para el ingreso.

"Honestamente, no sé desde cuándo. Solo recuerdo que al momento de mi ingreso al sistema científico de CONICET, conocí investigadores sin $\mathrm{PhD}$ y fui dirigida por una persona que no tenía doctorado. Personalmente, no estoy de acuerdo. Creo que para dirigir un doctorado, hay que tener la experiencia personal de haberlo realizado", Directora Mujer 1.

El énfasis de la Directora Mujer 2 estuvo puesto en la capacidad de la persona para abrir líneas nuevas de investigación tanto en la dirección puntual como al momento de publicar, pues para ella esto define quien tiene la aptitud. Ello, en virtud de que según sus propias palabras no es lo mismo iniciar una línea de trabajo que sumarse a la línea de otros investigadores.

"No dispongo de esa información", Directora Mujer 3.

Opina que la ausencia del título se puede suplir con publicaciones o son exigencias distintas. ¿Por qué?

"Considero que no son complementarios. Se puede publicar, hacer transferencia, o haber tenido una carrera de grado con un desempeño académico muy bueno o destacable y no necesariamente eso habilita para dirigir un doctorado. No se puede ayudar a transitar un camino que se desconoce. Hay etapas que se viven durante la ejecución del doctorado, inquietudes, proceso del pensamiento, técnicas, etc., que a mi criterio, sólo pueden conocerse si uno las vivió previamente", Directora Mujer 1.

En relación con la Directora Mujer 2, el aspecto esencial es el talento para abrir líneas nuevas de investigación.

"No tengo opinión formada al respecto", Directora Mujer 3.

¿La falta de publicaciones en inglés, en el marco de la CIC, es una debilidad?

"Si", Directora Mujer 1. 
"La falta de publicaciones en inglés, en el marco de la CIC, no es una debilidad", Directora Mujer 3.

¿Debe existir un compromiso moral de transferir a la sociedad los resultados de la investigación? ¿Por qué?

"Sí. Aunque esto pueda ocurrir a largo plazo, creo que un científico debe tener en algún lugar de sus proyecciones, la posibilidad de aportar ideas, potenciales soluciones a problemáticas de la sociedad, o contribuir a la apropiación de conocimiento, generando resultados originales e innovadores. Creo que es la mejor forma de demostrar que la inversión en CyT tiene un sentido y una razón de ser”, Directora Mujer 1.

Uno de los momentos más lindos de esta labor de investigación estuvo marcado por la charla con la Directora Mujer 2, pues fue impactante su nivel de compromiso y conocimiento sobre el desarrollo de la ciencia y tecnología. Una impronta claramente heredada de su madre (quien como asistente social en el Poder Judicial de Tucumán muchas veces llevó a niños en estado de vulnerabilidad a su casa hasta lograr su reinserción social) y también de su padre (quien fundó y fue durante muchos años el director del CITCA). Toda esa riqueza de su historia personal se percibe en la entonación de su voz y la evidente meditación de los temas que hacen a la coyuntura de su tarea diaria de investigación.

"El compromiso moral de transferir a la sociedad los resultados de la investigación ya existe, y se lleva a cabo", Directora Mujer 3.

\section{CONCLUSIONES}

Los PIO del CITCA (CONICET/UNCa) evidencian una mayoría de varones en su dirección, que claramente no encuentra justificación en la excelencia de su formación. En consecuencia, se ha corroborado la hipótesis base, esto es, "el reconocimiento difiere dependiendo si se es mujer o varón en la ciencia de Argentina". Así, en la dirección de los proyectos más importantes del organismo llama poderosamente la atención que, incluso, algunos varones no reúnen los requisitos mínimos, esto es, título de doctor (un caso) y categoría de independiente (en cuatro supuestos). Mientras que absolutamente todas las directoras mujeres poseen los requisitos para tal distinción. Indudablemente, al ser mujeres no se les permitiría ninguno de esos "beneficios", ya que ellas deben probar a cada paso su aptitud. Algo que, clara y socialmente, no se les exige a los varones como pre requisito de reconocimiento.

Las diferencias por género aparentemente no estarían tan marcadas a la hora de seleccionar postulantes a becas doctorales para comenzar la carrera, pero sí para promover a esas investigadoras. El problema es que estas diferencias no son explícitas sino simplemente que no instrumenta herramientas para la contención y 
permanencia de esas mujeres. A pesar de algunos logros la gran deuda pasada, presente y latente es el techo de cristal (o los clavos a los pies de las mujeres cuando tienen que cuidar a sus hijos e hijas o a sus padres o madres, o sufren acoso o violencia en laboratorios u hostigamiento en territorios descampados y machistas) pero la discriminación es clara. Empiezan más mujeres que varones cuando el sueldo es bajo y las responsabilidades son de principiantes y terminan más varones que mujeres cuando el sueldo y el poder son altos.

"La creciente presencia de mujeres sobre el total de investigadores/as muestra importantes diferencias según las categorías de la carrera de Investigador/a Científico/a. Mientras que las mujeres constituyen una clara mayoría en las etapas iniciales de la carrera (asistentes y adjuntos/as), su presencia disminuye sistemáticamente en la medida que se progresa en la carrera, hasta el punto que constituyen apenas el 25 por ciento de los investigadores/as superiores, el tope de la carrera”, remarca la química Ana Franchi, Investigadora del CONICET, directora del Centro de Estudios Farmacológicos y Botánicos (Cefybo) y presidenta de la Red Argentina de Genero, Ciencia y Tecnología (RAGCyT)

En consecuencia, pese al bonito dato de color de que cada vez más mujeres acceden por, puro y exclusivo, merito al conocimiento; según se evidencia una vez más a lo largo de esta investigación en curso, coloca a quien lee datos parciales en el riesgo de celebrar el árbol que esconde un bosque en problemas detrás de sí. Hecho que, lamentablemente, invita a concluir que no se pudo corroborar el auspicioso pronóstico de la destacada especialista María Elina Estébanez, quien sostuvo en su informe de 2002 que: la proporción significativa de becarias permitía avizorar su proyección institucional, "porque no solo se refiere a la presencia actual de mujeres en las actividades de investigación, sino que permite conjeturar que la cantidad total de investigadores tenderá a revertir la tendencia en cuanto a la participación por sexo en el futuro" (ESTÉBANEZ, 2002: 92)

"Conocer la historia de la ciencia es reconocer la mortalidad de cualquier pretensión de verdad universal. Cualquier visión pasada de verdad científica, cualquier modelo de los fenómenos naturales, con el tiempo ha resultado ser más limitado de lo que pretendían sus defensores. La supervivencia de la diferencia productiva en la ciencia requiere que situemos todas las pretensiones de hegemonía intelectual en su lugar adecuado que se entienda que tales pretensiones, por su misma naturaleza, son más políticas que científicas" (FOX-KELLER, 2001: 153)

\section{WOMEN, BY WOMEN OF SCIENCE IN ARGENTINA}

\section{Abstract}

In Argentina, six out of every ten scholarship holders of the Consejo Nacional de Investigaciones Científicas y 
Técnicas (CONICET) are women. However, the female majority that occurs at the beginning of the career is reversed markedly to reach positions of hierarchy, so the initial 75 percent only 25 percent of women remain. Consequently, the attribution of higher categories is not neutral to gender dynamics, since the participation of women in Science, Technology and Industry reproduces the forms of oppression of women that exist globally; to such an extent that similar, and even identical, academic and professional careers are provided in a different way if one is male or female. Therefore, the taxonomy focuses on the trajectories of the five women compared to those of the seven men, who are the current directors of the Proyectos de Investigación Orientados (PIO) del Centro de Investigación y Transferencia de Catamarca (CITCA) by resolution 2049/16.

Keywords: Sexual Dimorphism, Gender, CONICET and Argentina.

\section{REFERENCIAS BIBLIOGRÁFICAS}

ALBORNOZ, M.; BARRERE, R.; BAGENETA, M.; CHARREAU, H.; LÓPEZ MONRROY, E. E.; MATAS, L. (2008) Nanotecnología: Tendencias recientes en I+D. Argentina en el contexto internacional; disponible en www. caicyt.gov.ar

ALSINA, F. (2011) Investigación, transferencia, tecnología. En el pensamiento latinoamericano en la problemática ciencia-tecnología-desarrollo-dependencia. - la ed. - Buenos Aires: Ediciones Biblioteca Nacional.

ARAYA UMAÑA, S. (2014) La categoría analítica de género: notas para un debate. HALLAZGOS, ISSN: 1794-3841. Año 12, No 23. Bogotá, D. C., Universidad Santo Tomás, pp. 287-305.

ARRIGHI, A. (2012) Dimorfismo sexual humano. Revista de la Asociación Médica Argentina, Vol. 125 Número 1.

BAYER G. (2011) Autonomía nacional y política científica y tecnológica. En el pensamiento latinoamericano en la problemática ciencia-tecnología-desarrollo-dependencia. - la ed. - Buenos Aires: Ediciones Biblioteca Nacional.

BOX-STEFFENSMEIR, JM, CUNHA, RC. VARBANOV, RA. , HOH, S., KNISLEY, ML. and HOLMES, MA. (2015) Survival Analysis of Faculty Retention and Promotion in the Social Sciences by Gender. PLoS ONE10(11):e0143093.doi:10.1371/journal. pone.0143093

BLUM, D. (1997) Sex on the Brain: The Biological Differences between Men and Women. Viking Press.

CHANG CASTILLO, H. (2010) El modelo de la triple hélice como medio para la vinculación entre universidad y empresa, Revista Nacional de Administración, Costa Rica.

CODNER, D. G., BECERRA, P., \& DÍAZ, A. (2012) Blind Technology Transfer or Technological Knowledge Leakage: a Case Study from the South. Journal of technology management \& innovation, 7(2), $184-$ 195. 
CORIAT, B. y ORSI, F. (2005) Are strong patents beneficial to innovative activities, Industrial and Corporate Change, Volume 14, Number 6.

ESTÉBANEZ, M. E. y LÁSCARIS COMNENO, T. (2004) La mujer y la ciencia en Centroamérica. Un ejercicio de aplicación del enfoque de género en la construcción de indicadores. En: El Estado de la Ciencia 2003. Principales indicadores de ciencia y tecnología Iberoamericanos/ Interamericanos RICYT. CYTED REDES. Buenos Aires.

ESTÉBANEZ, Ma. E., SERIAL, A., de FILIPPO, D. (2003) Participación de la mujer en el sistema de Investigación y desarrollo en Argentina. Informe del caso argentino. Programa GENTEC-UNESCO-OEI. Documento de trabajo No 8 centro Redes. ISSN 2313 - 9811. En: www.centroredes.org.ar/documentos/files/Doc.Nro8.pdf

FERNÁNDEZ RIUS, L. (2010) Género y ciencia, o, ¿La apoteosis del egoísmo? La Habana, Cuba: Editorial de la Mujer.

FIELDING, N. and FIELDING, J. (1986) Linking Data: the Articulation of Qualitative and Quantitative Methods in Social Research, Beverly Hills, London, Stage.

FOX-KELLER, E. (2001) Reflexiones sobre género y sexo.

FRANCHI, A.; ATRIO, J.; MAFFIA D. y KOCHEN S. (2008) Inserción de las Mujeres en el Sector Científico-Tecnológico en la Argentina (1984-2006), ARBOR CLXXXIV 733 septiembre-octubre, 827-834.

GEARY, D. (1998) Male, Female: The Evolution of Human Sex Differences. American Psychological Association.

KAMINSKI, D. and GEISLE, C. (2012) Survival Analysis of Faculty Retention in Science and Engineering by Gender. DOI: 10.1126/science.1214844

KIMURA, D. (1992) Sex Differences in the Brain. Scientific American, Vol. 267, No. 3, pages 118- 125.

LAMAS, M. (1999) Usos, dificultades y posibilidades de la categoría género. Papeles de Población, vol. 5, núm. 21. Universidad Autónoma del Estado de México Toluca, México, julio-septiembre, pp. 147-178.

LAMAS, M. (1999) Usos, dificultades y posibilidades de la categoría género. Papeles de Población, vol. 5, núm. 21. Universidad Autónoma del Estado de México Toluca, México, julio-septiembre, pp. 147-178.

LARRY C. (2005) His Brain, Her Brain. Scientific American, Vol. 292, No. 5, pp. 40-47 Published by: Scientific American, a division of Nature America, Inc. Stable URL: http://www.jstor.org/stable/26060993

LÓPEZ MONRROY, E. E. (2014) Política de la Innovación Inclusiva, Editorial de la Universidad Nacional de Catamarca, ISBN: 978-987-661-185-5. 
LÓPEZ MONRROY, E. E. (2018) El Rol Actual de la Mujer en la Innovación de Argentina, Revista Hermenéutica, Río Negro (Santa Cruz)

PÉREZ SELDEÑO, E. (2000) Institucionalización de la Ciencia: valores epistémicos y contextuales. Un caso ejemplar. En Cadernos Pagu (15), Madrid (España)

PRESTON, A. (2005) Leaving Science in the 1990s. Russell Sage Foundation, 2004. Working Paper Haverford College, 2005.

ROWBOTHAM, S. (1973) Woman's consciousness, man's world. Harmondsworth: Penguin Press, p. 28.8.

TORRES SALINAS, D., MUÑOZ MUÑOZ, A. M. y JIMÉNEZ CONTRERAS, E. (2011) Análisis bibliométrico de la situación de las mujeres investigadoras de Ciencias Sociales y Jurídicas en España. Revista Española de Documentación Científica, 34, 1, enero-marzo, 11-28, ISSN: 0210-0614.

VERA, M. C. (2005) Mujeres Latinoamericanas: Su inserción en los estudios superiores y en el campo de la investigación cientifica, Rhec.

Trabalho enviado em 08 de maio de 2018.

Aceito em 24 de julho de 2018. 